\title{
Kinetic Equation for Soliton Gas: Integrable Reductions
}

\author{
E.V. Ferapontov ${ }^{1,2}$ (I) $\cdot$ M.V. Pavlov ${ }^{3}$
}

Received: 27 September 2021 / Accepted: 4 February 2022 / Published online: 4 March 2022

(c) The Author(s) 2022

\begin{abstract}
Macroscopic dynamics of soliton gases can be analytically described by the thermodynamic limit of the Whitham equations, yielding an integro-differential kinetic equation for the density of states. Under a delta-functional ansatz, the kinetic equation for soliton gas reduces to a non-diagonalisable system of hydrodynamic type whose matrix consists of several $2 \times 2$ Jordan blocks. Here we demonstrate the integrability of this system by showing that it possesses a hierarchy of commuting hydrodynamic flows and can be solved by an extension of the generalised hodograph method. Our approach is a generalisation of Tsarev's theory of diagonalisable systems of hydrodynamic type to quasilinear systems with non-trivial Jordan block structure.
\end{abstract}

Keywords Soliton gas · Kinetic equation · Hydrodynamic reduction · Commuting flow $\cdot$ Conservation law $\cdot$ Generalised hodograph formula

Mathematics Subject Classification 35Q51 $\cdot 35 \mathrm{Q} 83 \cdot 37 \mathrm{~K} 10$

Communicated by Peter Miller.

$\bowtie \quad$ E.V. Ferapontov

E.V.Ferapontov@1boro.ac.uk

M.V. Pavlov

M.V.Pavlov@lboro.ac.uk

1 Department of Mathematical Sciences, Loughborough University, Loughborough, Leicestershire LE11 3TU, UK

2 Institute of Mathematics, Ufa Federal Research Centre, Russian Academy of Sciences, 112, Chernyshevsky Street, Ufa, Russia 450077

3 Institute of Physics, Mathematics and Informational Technology, Immanuel Kant Baltic Federal University, Al. Nevsky St. 14, Kaliningrad, Russia 236041 


\section{Introduction}

Quasilinear systems of the form

$$
u_{t}=V(u) u_{x}
$$

have been thoroughly investigated in the literature. Here $u=\left(u^{1}, \ldots, u^{n}\right)^{T}$ is a column vector of the dependent variables and $V$ is a $n \times n$ matrix. The main emphasis has always been on the strictly hyperbolic case where the matrix $V$ has real distinct eigenvalues. Under the additional condition that the Haantjes tensor of matrix $V$ vanishes, any such system can be reduced to a diagonal form,

$$
r_{t}^{i}=v^{i}(r) r_{x}^{i}
$$

$i=1, \ldots, n$, in specially adapted coordinates $r^{1}, \ldots, r^{n}$ known as Riemann invariants. We recall that the Haantjes tensor $H_{j k}^{i}$ of matrix $V=\left\{v_{j}^{i}(u)\right\}$ is defined by the formula

$$
H_{j k}^{i}=N_{p r}^{i} v_{j}^{p} v_{k}^{r}+N_{j k}^{p} v_{r}^{i} v_{p}^{r}-N_{j r}^{p} v_{p}^{i} v_{k}^{r}-N_{r k}^{p} v_{p}^{i} v_{j}^{r}
$$

where $N_{j k}^{i}$ is the Nijenhuis tensor,

$$
N_{j k}^{i}=v_{j}^{s} \partial_{u^{s}} v_{k}^{i}-v_{k}^{s} \partial_{u^{s}} v_{j}^{i}-v_{s}^{i}\left(\partial_{u^{j}} v_{k}^{s}-\partial_{u^{k}} v_{j}^{s}\right)
$$

Systems of type (2) govern a wide range of problems in pure and applied mathematics, see, e.g., (Tsarev 1985, 1991; Dubrovin and Novikov 1989; Serre 1999, 2000; Sévennec 1994). It was shown by Tsarev $(1985,1991)$ that under the so-called semi-Hamiltonian constraint,

$$
\left(\frac{v_{r^{j}}^{i}}{v^{j}-v^{i}}\right)_{r^{k}}=\left(\frac{v_{r^{k}}^{i}}{v^{k}-v^{i}}\right)_{r^{j}},
$$

system (2) possesses infinitely many commuting flows and conservation laws and can be solved by the generalised hodograph method (here $i \neq j \neq k$ and $v_{r^{j}}^{i}=\partial v^{i} / \partial r^{j}$, etc). Recall that commuting flows of diagonal systems (2) are also diagonal,

$$
r_{y}^{i}=w^{i}(r) r_{x}^{i}
$$

where the requirement of commutativity, $r_{t y}^{i}=r_{y t}^{i}$, implies the relations $(i \neq j)$ :

$$
\frac{v_{r^{j}}^{i}}{v^{j}-v^{i}}=\frac{w_{r^{j}}^{i}}{w^{j}-w^{i}} .
$$


Introducing the notation $a_{i j}=\frac{v_{r j}^{i}}{v^{j}-v^{i}}$ and rewriting the equations for commuting flows in the form

$$
w_{r^{j}}^{i}=a_{i j}\left(w^{j}-w^{i}\right)
$$

the requirement of their compatibility, $\left(w_{r^{j}}^{i}\right)_{r^{k}}=\left(w_{r^{k}}^{i}\right)_{r^{j}}$, implies the integrability conditions

$$
a_{i j, r^{k}}=a_{i j} a_{j k}+a_{i k} a_{k j}-a_{i j} a_{i k}
$$

which are equivalent to the semi-Hamiltonian property (3). Under conditions (5), system (4) for commuting flows possesses infinitely many solutions parametrised by $n$ arbitrary functions of one variable (Tsarev 1985).

In this paper, we study integrability aspects of quasilinear systems (1) whose matrix $V$ consists of $n$ Jordan blocks of size $2 \times 2$ :

$$
\begin{aligned}
& r_{t}^{i}=v^{i} r_{x}^{i}+p^{i} \eta_{x}^{i} \\
& \eta_{t}^{i}=v^{i} \eta_{x}^{i}
\end{aligned}
$$

$i=1, \ldots, n$, where the coefficients $v^{i}(r, \eta)$ and $p^{i}(r, \eta)$ are functions of the $2 n$ dependent variables $r=\left(r^{1}, \ldots, r^{n}\right)$ and $\eta=\left(\eta^{1}, \ldots, \eta^{n}\right)$. Systems of this form have appeared recently as delta-functional reductions in the kinetic equation for soliton gas (Pavlov et al. 2012), see below, as well as hydrodynamic reductions in linearly degenerate dispersionless integrable systems in multidimensions, see Sect. 5.2. In Sect. 2, we derive Jordan block analogues of equations for commuting flows (4) and integrability conditions (5). Although obtained in exactly the same way as for diagonalisable systems, these conditions are considerably more complicated.

Block-diagonal systems of type (6) share the following general properties:

- The Haantjes tensor of system (6) vanishes identically, which makes these systems natural parabolic analogues of diagonal systems (2). Note that systems (2) can be obtained from (6) by setting $\eta^{i}=$ const.

- The class of systems (6) is invariant under changes of variables of the form

$$
r^{i} \rightarrow R^{i}\left(r^{i}, \eta^{i}\right), \quad \eta^{i} \rightarrow \mathcal{N}^{i}\left(\eta^{i}\right)
$$

where $R^{i}$ and $\mathcal{N}^{i}$ are arbitrary functions of the indicated arguments. Note that the group preserving the class of diagonal systems (2) is more narrow, generated by transformations of the form $r^{i} \rightarrow R^{i}\left(r^{i}\right)$, functions of one variable only.

- Matrices $V$ corresponding to systems (6) form a commutative family. In particular, hydrodynamic commuting flows of systems (6) are also of type (6). This observation allows one to develop the integrability theory of such systems in full analogy with Tsarev's theory of diagonalisable systems (2) (Tsarev 1985, 1991), by requiring the existence of a hierarchy of commuting flows. 
Remark Integrable quasilinear systems of a single Jordan block type (of arbitrary size) have appeared in the literature as degenerations of hydrodynamic systems associated with multi-dimensional hypergeometric functions (Kodama and Konopelchenko 2016), in the context of parabolic regularisation of the Riemann equation (Konopelchenko and Ortenzi 2018) and as reductions in hydrodynamic chains and linearly degenerate dispersionless PDEs in 3D (Pavlov 2018). A connection of such systems with the modified KP hierarchy was established in Xue and Ferapontov (2020).

Our interest in systems (6) stems from the study of El's integro-differential kinetic equation for dense soliton gas (El 2003; El and Kamchatnov 2005; El et al. 2011):

$$
\begin{gathered}
f_{t}+(s f)_{x}=0, \\
s(\eta)=S(\eta)+\int_{0}^{\infty} G(\mu, \eta) f(\mu)[s(\mu)-s(\eta)] d \mu,
\end{gathered}
$$

where $f(\eta)=f(\eta, x, t)$ is the distribution function and $s(\eta)=s(\eta, x, t)$ is the associated transport velocity. Here the variable $\eta$ is a spectral parameter in the Lax pair associated with the dispersive hydrodynamics; the function $S(\eta)$ (free soliton velocity) and the kernel $G(\mu, \eta)$ (phase shift due to pairwise soliton collisions) are independent of $x$ and $t$. The kernel $G(\mu, \eta)$ is assumed to be symmetric: $G(\mu, \eta)=G(\eta, \mu)$. Equation (8) describes the evolution of a dense soliton gas and represents a broad generalisation of Zakharov's kinetic equation for rarefied soliton gas (Zakharov 1971). It has appeared independently in the context of generalised hydrodynamics of multibody quantum integrable systems (Doyon and Yoshimura 2018). Note that both $S(\eta)$ and $G(\mu, \eta)$ are system specific. In the special case

$$
S(\eta)=4 \eta^{2}, \quad G(\mu, \eta)=\frac{1}{\eta \mu} \log \left|\frac{\eta-\mu}{\eta+\mu}\right|,
$$

system (8) was derived in El (2003) as thermodynamic limit of the KdV Whitham equations and generalised in El and Kamchatnov (2005), El and Tovbis (2020) to the NLS case. It was demonstrated in Pavlov et al. (2012) that under a delta-functional ansatz,

$$
f(\eta, x, t)=\sum_{i=1}^{n} u^{i}(x, t) \delta\left(\eta-\eta^{i}(x, t)\right),
$$

system (8) reduces to a $2 n \times 2 n$ quasilinear system for $u^{i}(x, t)$ and $\eta^{i}(x, t)$,

$$
u_{t}^{i}=\left(u^{i} v^{i}\right)_{x}, \quad \eta_{t}^{i}=v^{i} \eta_{x}^{i}
$$

where $v^{i} \equiv-s\left(\eta^{i}, x, t\right)$ can be recovered from the linear system

$$
v^{i}=-S\left(\eta^{i}\right)+\sum_{k \neq i} \epsilon^{k i} u^{k}\left(v^{k}-v^{i}\right), \quad \epsilon^{k i}=G\left(\eta^{k}, \eta^{i}\right), k \neq i .
$$


To make the paper more self-contained, we sketch the derivation of Eqs. (10), (11) in Appendix. The special choice $\eta^{i}(x, t)=$ const was discussed previously in El et al. (2011). In this case, the last $n$ equations (10) are satisfied identically, while the first $n$ equations constitute an integrable diagonalisable linearly degenerate system. The main aim of this paper is to demonstrate integrability of the full system (10). This task is not so straightforward since system (10) is not diagonalisable; thus, Tsarev's theory of diagonalisable systems of hydrodynamic type (Tsarev 1985, 1991, see also Dubrovin and Novikov 1989) does not apply, and a suitable generalisation is required. Following (Pavlov et al. 2012), let us introduce the new variables $r^{i}$ by the formula

$$
r^{i}=-\frac{1}{u^{i}}\left(1+\sum_{k \neq i} \epsilon^{k i} u^{k}\right)
$$

In the dependent variables $r^{i}, \eta^{i}$, system (10) reduces to block-diagonal form (6) where the coefficients $v^{i}$ and $p^{i}$ can be expressed in terms of $(r, \eta)$-variables as follows. Let us introduce the $n \times n$ matrix $\hat{\epsilon}$ with diagonal entries $r^{1}, \ldots, r^{n}$ (so that $\epsilon^{i i}=r^{i}$ ) and off-diagonal entries $\epsilon^{i k}=G\left(\eta^{i}, \eta^{k}\right), k \neq i$. Note that this matrix is symmetric due to the symmetry of the kernel $G$. Define another symmetric matrix $\hat{\beta}=-\hat{\epsilon}^{-1}$. Explicitly, for $n=2$ we have

$$
\hat{\epsilon}=\left(\begin{array}{cc}
r^{1} & \epsilon^{12} \\
\epsilon^{12} & r^{2}
\end{array}\right), \quad \hat{\beta}=\frac{1}{r^{1} r^{2}-\left(\epsilon^{12}\right)^{2}}\left(\begin{array}{cc}
-r^{2} & \epsilon^{12} \\
\epsilon^{12} & -r^{1}
\end{array}\right) .
$$

Denote $\beta_{i k}$ the matrix elements of $\hat{\beta}$ (indices $i$ and $k$ are allowed to coincide). Introducing the notation $\xi^{k}\left(\eta^{k}\right)=-S\left(\eta^{k}\right)$, we have the following formulae for $u^{i}, v^{i}$ and $p^{i}$ (Pavlov et al. 2012):

$$
u^{i}=\sum_{k=1}^{n} \beta_{k i}, \quad v^{i}=\frac{1}{u^{i}} \sum_{k=1}^{n} \beta_{k i} \xi^{k}, \quad p^{i}=\frac{1}{u^{i}}\left(\sum_{k=1}^{n} \epsilon_{, \eta^{i}}^{k i}\left(v^{k}-v^{i}\right) u^{k}+\left(\xi^{i}\right)^{\prime}\right),
$$

see Sect. 5.1 for explicit form of $v^{i}$ and $p^{i}$ in the simplest cases $n=1,2$. Here and in what follows we use the notation $\epsilon_{, \eta^{i}}^{k i}$ to indicate partial derivative with respect to $\eta^{i}$. Although the explicit form of $v^{i}$ and $p^{i}$ gets increasingly more complicated as $n$ grows, there exists a remarkably compact formula for the general solution of system (6) corresponding to reduction (10) that works for arbitrary $n$ :

$$
r^{i}=\frac{\varphi_{, \eta^{i}}^{i}-\left(\xi^{i}\right)^{\prime} t}{\mu^{i}}, \quad \varphi^{i}\left(\eta^{1}, \ldots, \eta^{n}\right)=x+\xi^{i}\left(\eta^{i}\right) t
$$

here $\mu^{i}\left(\eta^{i}\right)$ are arbitrary functions of their arguments and the functions $\varphi^{i}\left(\eta^{1}, \ldots, \eta^{n}\right)$ satisfy the relations $\varphi_{, \eta^{j}}^{i}=\epsilon^{j i}\left(\eta^{i}, \eta^{j}\right) \mu^{j}\left(\eta^{j}\right), i \neq j$, no summation. The last $n$ 
equations (13) define $\eta^{i}(x, t)$ as implicit functions of $x$ and $t$; then, the first $n$ equations define $r^{i}(x, t)$ explicitly. Formula (13) results from the generalised hodograph approach outlined in Sect. 4.

We demonstrate integrability of system (10) by explicitly presenting its commuting flows (Sect. 2) and conservation laws (Sect. 3) parametrised by $2 n$ arbitrary functions of one variable. We emphasise that integrability of system (10) holds for arbitrary functions $S(\eta)$ and $G(\mu, \eta)$ in the kinetic equation (8), even without the assumption of symmetry of the kernel $G$. Finally, we point out that formula (13) does not possess any obvious limit to the case $\eta^{i}=$ const discussed in El et al. (2011). The main reason for this is the structure of commuting flows of systems (6), see Sect. 2.2.

\section{Commuting Flows}

Given system (6), let us look for commuting flows in the form

$$
\begin{aligned}
r_{y}^{i} & =w^{i} r_{x}^{i}+q^{i} \eta_{x}^{i}, \\
\eta_{y}^{i} & =w^{i} \eta_{x}^{i} .
\end{aligned}
$$

The requirement of commutativity of (6) and (14), $r_{t y}^{i}=r_{y t}^{i}$ and $\eta_{t y}^{i}=\eta_{y t}^{i}$, leads to the two groups of conditions. First of all, for every $i \in\{1, \ldots, n\}$ one has the relations

$$
\begin{gathered}
\frac{v_{r^{i}}^{i}}{p^{i}}=a_{i}=\frac{w_{r^{i}}^{i}}{q^{i}}, \\
\frac{v_{\eta^{i}}^{i}-p_{r^{i}}^{i}}{p^{i}}=b_{i}=\frac{w_{\eta^{i}}^{i}-q_{r^{i}}^{i}}{q^{i}} .
\end{gathered}
$$

Secondly, for every $i \neq j \in\{1, \ldots, n\}$ one has the relations

$$
\begin{aligned}
\frac{v_{r j}^{i}}{v^{j}-v^{i}} & =a_{i j}=\frac{w_{r^{j}}}{w^{j}-w^{i}}, \\
\frac{v_{\eta^{j}}^{i}-a_{i j} p^{j}}{v^{j}-v^{i}} & =b_{i j}=\frac{w_{\eta^{j}}^{i}-a_{i j} q^{j}}{w^{j}-w^{i}}, \\
\frac{p_{r^{j}}^{i}+a_{i j} p^{i}}{v^{j}-v^{i}} & =c_{i j}=\frac{q_{r j}^{i}+a_{i j} q^{i}}{w^{j}-w^{i}}, \\
\frac{p_{\eta^{j}}^{i}+b_{i j} p^{i}-c_{i j} p^{j}}{v^{j}-v^{i}} & =d_{i j}=\frac{q_{\eta^{j}}^{i}+b_{i j} q^{i}-c_{i j} q^{j}}{w^{j}-w^{i}} .
\end{aligned}
$$

Here $a_{i}, b_{i}, a_{i j}, b_{i j}, c_{i j}, d_{i j}$ in (15) and (16) is just the notation for the coefficients shared by commuting flows of the hierarchy. Commutativity conditions (15), (16) are presented in symmetric form where the left-hand sides (respectively, right-hand sides) are expressed in terms of system (6) (respectively, its commuting flow (14)). 


\subsection{Integrability Conditions}

Integrability conditions of system (6) can be obtained by calculating consistency conditions of the linear system (15), (16) governing commuting flows. Thus, we regard $v^{i}, p^{i}$ as given and $w^{i}, q^{i}$ as the unknowns. First of all, for every $i \in\{1, \ldots, n\}$ one has the relations

$$
w_{r^{i}}^{i}=a_{i} q^{i}, \quad w_{\eta^{i}}^{i}=q_{r^{i}}^{i}+b_{i} q^{i}
$$

Secondly, for every $i \neq j \in\{1, \ldots, n\}$ one has the relations

$$
\begin{gathered}
w_{r^{j}}^{i}=a_{i j}\left(w^{j}-w^{i}\right), \quad w_{\eta^{j}}^{i}=a_{i j} q^{j}+b_{i j}\left(w^{j}-w^{i}\right), \\
q_{r^{j}}^{i}=-a_{i j} q^{i}+c_{i j}\left(w^{j}-w^{i}\right), \quad q_{\eta^{j}}^{i}=-b_{i j} q^{i}+c_{i j} q^{j}+d_{i j}\left(w^{j}-w^{i}\right) .
\end{gathered}
$$

Note that the relations $\left(w_{r^{i}}^{i}\right)_{\eta^{i}}=\left(w_{\eta^{i}}^{i}\right)_{r^{i}}$ give second-order derivatives $q_{r^{i} r^{i}}^{i}$ :

$$
q_{r^{i} r^{i}}^{i}=a_{i} q_{\eta^{i}}^{i}-b_{i} q_{r^{i}}^{i}+\left(a_{i, \eta^{i}}-b_{i, r^{i}}\right) q^{i}
$$

Expressed in terms of the coefficients $a_{i}, b_{i}, a_{i j}, b_{i j}, c_{i j}, d_{i j}$, the consistency conditions of relations (17), (18) and (19) are the required integrability conditions for system (6). There are two types of integrability conditions, involving two and three distinct indices, respectively.

Two-index conditions There are several groups thereof, each involving two distinct indices $i \neq j$.

The consistency condition $\left(w_{r^{i}}^{i}\right)_{r^{j}}=\left(w_{r^{j}}^{i}\right)_{r^{i}}$ implies

$$
a_{i, r^{j}}=0, \quad a_{i j, r^{i}}=a_{i j} a_{j i}+a_{i} c_{i j}
$$

The consistency condition $\left(w_{r^{i}}^{i}\right)_{\eta^{j}}=\left(w_{\eta^{j}}^{i}\right)_{r^{i}}$ implies

$$
a_{i, \eta^{j}}=0, \quad b_{i j, r^{i}}=b_{i j} a_{j i}+a_{i j} c_{j i}+a_{i} d_{i j}
$$

The consistency condition $\left(w_{\eta^{i}}^{i}\right)_{r^{j}}=\left(w_{r^{j}}^{i}\right)_{\eta^{i}}$ implies

$$
b_{i, r^{j}}=2 a_{i j} a_{j i}+2 a_{i} c_{i j}, \quad a_{i j, \eta^{i}}=a_{i j} b_{j i}-c_{i j} a_{j i}+b_{i} c_{i j}+c_{i j, r^{i}}
$$

The consistency condition $\left(w_{\eta^{i}}^{i}\right)_{\eta^{j}}=\left(w_{\eta^{j}}^{i}\right)_{\eta^{i}}$ implies

$$
\begin{aligned}
& b_{i, \eta^{j}}=2 a_{i j} c_{j i}+2 b_{i j} a_{j i}+2 a_{i} d_{i j}, \\
& b_{i j, \eta^{i}}=b_{i j} b_{j i}+a_{i j} d_{j i}-d_{i j} a_{j i}-c_{i j} c_{j i}+b_{i} d_{i j}+d_{i j, r^{i}} .
\end{aligned}
$$


The consistency condition $\left(w_{r^{j}}^{i}\right)_{\eta^{j}}=\left(w_{\eta^{j}}^{i}\right)_{r^{j}}$ implies

$$
a_{i j, r^{j}}=b_{j} a_{i j}-a_{j} b_{i j}-a_{i j}^{2}, \quad a_{i j, \eta^{j}}=b_{i j, r^{j}} .
$$

The consistency condition $\left(q_{r^{j}}^{i}\right)_{\eta^{j}}=\left(q_{\eta^{j}}^{i}\right)_{r^{j}}$ implies

$$
c_{i j, r^{j}}=b_{j} c_{i j}-a_{j} d_{i j}-2 a_{i j} c_{i j}, \quad c_{i j, \eta^{j}}=d_{i j, r^{j}}
$$

Finally, direct calculation shows that the remaining consistency conditions between (19) and the last two equations (18), namely, $\left(q_{r^{j}}^{i}\right)_{r^{i} r^{i}}=\left(q_{r^{i} r^{i}}^{i}\right)_{r^{j}}$ and $\left(q_{\eta^{j}}^{i}\right)_{r^{i} r^{i}}=$ $\left(q_{r^{i} r^{i}}^{i}\right)_{\eta^{j}}$, are satisfied identically. Relations (20)-(25) form a complete set of integrability conditions for $4 \times 4$ systems (6) (case of two Jordan blocks). For more than two Jordan blocks, additional three-index conditions are required.

Three-index conditions There are several groups thereof, each involving three distinct indices $i \neq j \neq k$.

The consistency condition $\left(w_{r^{j}}^{i}\right)_{r^{k}}=\left(w_{r^{k}}^{i}\right)_{r^{j}}$ implies

$$
a_{i j, r^{k}}=a_{i j} a_{j k}+a_{i k} a_{k j}-a_{i j} a_{i k} .
$$

The consistency condition $\left(w_{r^{j}}^{i}\right)_{\eta^{k}}=\left(w_{\eta^{k}}^{i}\right)_{r^{j}}$ implies

$$
\begin{aligned}
& a_{i j, \eta^{k}}=a_{i j} b_{j k}+a_{i k} c_{k j}+b_{i k} a_{k j}-a_{i j} b_{i k}, \\
& b_{i j, r^{k}}=b_{i j} a_{j k}+a_{i k} b_{k j}+a_{i j} c_{j k}-a_{i k} b_{i j} .
\end{aligned}
$$

The consistency condition $\left(w_{\eta^{j}}^{i}\right)_{\eta^{k}}=\left(w_{\eta^{k}}^{i}\right)_{\eta^{j}}$ implies

$$
b_{i j, \eta^{k}}=a_{i j} d_{j k}+a_{i k} d_{k j}+b_{i j} b_{j k}+b_{i k} b_{k j}-b_{i j} b_{i k} .
$$

The consistency condition $\left(q_{r^{j}}^{i}\right)_{r^{k}}=\left(q_{r^{k}}^{i}\right)_{r^{j}}$ implies

$$
c_{i j, r^{k}}=c_{i j} a_{j k}+c_{i k} a_{k j}-c_{i j} a_{i k}-c_{i k} a_{i j} .
$$

The consistency condition $\left(q_{r^{j}}^{i}\right)_{\eta^{k}}=\left(q_{\eta^{k}}^{i}\right)_{r^{j}}$ implies

$$
\begin{aligned}
& c_{i j, \eta^{k}}=c_{i j} b_{j k}+c_{i k} c_{k j}+d_{i k} a_{k j}-a_{i j} d_{i k}-c_{i j} b_{i k} \\
& d_{i j, r^{k}}=d_{i j} a_{j k}+c_{i j} c_{j k}+c_{i k} b_{k j}-a_{i k} d_{i j}-c_{i k} b_{i j} .
\end{aligned}
$$

The consistency condition $\left(q_{\eta^{j}}^{i}\right)_{\eta^{k}}=\left(q_{\eta^{k}}^{i}\right)_{\eta^{j}}$ implies

$$
d_{i j, \eta^{k}}=c_{i j} d_{j k}+c_{i k} d_{k j}+d_{i j} b_{j k}+d_{i k} b_{k j}-b_{i j} d_{i k}-b_{i k} d_{i j}
$$

Relations (20)-(31) form a complete set of integrability conditions for systems (6). They can be viewed as Jordan block analogues of the semi-Hamiltonian property (5). 
For integrable systems (6), the general solution of equations $(17)-(19)$ for commuting flows depends on $2 n$ arbitrary functions of one variable.

For system (10) governing reductions in the soliton gas equation, all integrability conditions can be verified by direct calculation (which is quite cumbersome, see Sect. 5.1). In fact, in this particular case commuting flows can be found explicitly: the general commuting flow (14) of system (6) corresponding to reduction (10) has the coefficients

$$
w^{i}=\frac{1}{u^{i}} \sum_{k=1}^{n} \beta_{k i} \varphi^{k}, \quad q^{i}=\frac{1}{u^{i}}\left(\sum_{k=1}^{n} \epsilon_{, \eta^{i}}^{k i}\left(w^{k}-w^{i}\right) u^{k}-r^{i} \mu^{i}+\varphi_{, \eta^{i}}^{i}\right)
$$

where $\mu^{i}\left(\eta^{i}\right)$ are $n$ arbitrary functions of one variable and the functions $\varphi^{i}\left(\eta^{1}, \ldots, \eta^{n}\right)$ satisfy the relations $\partial_{\eta^{j}} \varphi^{i}=\epsilon^{j i} \mu^{j}, \quad j \neq i$, no summation (same functions as in (13)). The general commuting flow depends on $2 n$ arbitrary functions of one variable: $n$ functions $\mu^{i}\left(\eta^{i}\right)$, plus extra $n$ functions coming from $\varphi^{i}$. This demonstrates integrability of the system in question.

\subsection{Genuinely Nonlinear and Linearly Degenerate Systems}

The analysis of consistency conditions derived in Sect. 2.1 naturally splits into two cases, depending on whether the coefficients $a_{i}$ are zero or not. In view of the first relation (15), this is equivalent to $v_{r^{i}}^{i} \neq 0$ (genuine nonlinearity) or $v_{r^{i}}^{i}=0$ (linear degeneracy).

Genuinely nonlinear case $a_{i} \neq 0$. Keeping in mind the consistency conditions $a_{i, r^{j}}=$ $a_{i, \eta^{j}}=0$, one can set $a_{i}=1$ via a suitable equivalence transformation (7). In this case the first equation (17) implies $q^{i}=w_{r^{i}}^{i}$, so that the second equation (17) and the first two equations (18) take the form

$$
w_{\eta^{i}}^{i}=w_{r^{i} r^{i}}^{i}+b_{i} w_{r^{i}}^{i}, \quad w_{r^{j}}^{i}=a_{i j}\left(w^{j}-w^{i}\right), \quad w_{\eta^{j}}^{i}=a_{i j} w_{r^{j}}^{j}+b_{i j}\left(w^{j}-w^{i}\right),
$$

while the last two equations (18) will be satisfied identically due to the consistency conditions (20), (21). Thus, in the genuinely nonlinear case commuting flows can be parametrised in the form

$$
\begin{aligned}
r_{y}^{i} & =w^{i} r_{x}^{i}+w_{r^{i}}^{i} \eta_{x}^{i} \\
\eta_{y}^{i} & =w^{i} \eta_{x}^{i} .
\end{aligned}
$$

where $w^{i}$ solve the linear system (33). Note that the first equation (33) is the Lax operator of the modified KP (mKP) hierarchy. Thus, genuinely nonlinear integrable systems of type (14) are governed by the mKP hierarchy. The compatibility conditions of system (33) can be written in the form 


$$
\begin{aligned}
& a_{i j, r^{k}}=a_{i k} a_{k j}+a_{i j} a_{j k}-a_{i j} a_{i k}, \\
& a_{i j, \eta^{i}}=\partial_{r^{i}}\left(a_{i j, r^{i}}-2 a_{i j} a_{j i}+b_{i} a_{i j}\right)-a_{i j} b_{i, r^{i}}, \quad a_{i j, \eta^{j}}=b_{i j, r^{j}}, \quad a_{i j, \eta^{k}}=b_{i k, r^{j}}, \\
& b_{i, r^{j}}=2 a_{i j, r^{i}}, \quad b_{i, \eta^{j}}=2 b_{i j, r^{i}},
\end{aligned}
$$

where $b_{i j}=b_{j} a_{i j}-a_{i j}^{2}-a_{i j, r^{j}}$. This provides a compact formulation of integrability conditions (20)-(31).

Linearly degenerate case $a_{i}=0$. Then, the first relation (15) implies $w_{r^{i}}^{i}=0$; thus, commuting flows of linearly degenerate systems (6) are also linearly degenerate. Note that this is the case for system (10) governing reductions in the soliton gas equation. This explains the observation that formula (13) does not possess any obvious limit to the case $\eta^{i}=$ const ; indeed, in the diagonal case, generic commuting flows of linearly degenerate semi-Hamiltonian systems are not linearly degenerate.

For linearly degenerate systems, integrability conditions (20)-(31) constitute a Darboux-integrable system which can be 'solved explicitly', we hope to report the details elsewhere.

\section{Conservation Laws}

Conservation laws provide an alternative way to derive integrability conditions. Recall that conservation laws of system (6) are relations of the form

$$
h_{t}=g_{x}
$$

here $h(r, \eta)$ is the conserved density and $g(r, \eta)$ is the corresponding flux. The requirement that relation (34) holds identically modulo (6) implies the equations

$$
g_{r^{i}}=v^{i} h_{r^{i}}, \quad g_{\eta^{i}}=p^{i} h_{r^{i}}+v^{i} h_{\eta^{i}} .
$$

Eliminating the flux $g$, we obtain a system of second-order linear PDEs for the density $h$ :

$$
\begin{gathered}
h_{r^{i} r^{i}}=b_{i} h_{r^{i}}-a_{i} h_{\eta^{i}}, \\
h_{r^{i} r^{j}}=a_{i j} h_{r^{i}}+a_{j i} h_{r^{j}}, \\
h_{r^{i} \eta^{j}}=a_{j i} h_{\eta^{j}}+c_{j i} h_{r^{j}}+b_{i j} h_{r^{i}}, \\
h_{\eta^{i} \eta^{j}}=d_{i j} h_{r^{i}}+d_{j i} h_{r^{j}}+b_{i j} h_{\eta^{i}}+b_{j i} h_{\eta^{j}} ;
\end{gathered}
$$

here $i \neq j$ and the coefficients $a_{i}, b_{i}, a_{i j}, b_{i j}, c_{i j}, d_{i j}$ are the same as in Sect. 2, which confirms the well-known fact that commuting flows share the same conserved densities. One can show that the compatibility conditions of equations (35) coincide with integrability conditions (20)-(31) obtained in Sect. 2. For integrable systems (6), linear system (35) is in involution and its general solution $h$ depends on $2 n$ arbitrary functions of one variable. 
Remark In the genuinely nonlinear case, $a_{i}=1$, the first two equations (35) reduce to

$$
h_{\eta^{i}}=-h_{r^{i} r^{i}}+b_{i} h_{r^{i}}, \quad h_{r^{i} r^{j}}=a_{i j} h_{r^{i}}+a_{j i} h_{r^{j}},
$$

while the last two equations (35) will be satisfied identically due to integrability conditions (20)-(31). Thus, conserved densities $h$ are governed by the adjoint Lax pair (36) of the mKP hierarchy, compared with (33).

System (10) possesses infinitely many conservation laws of the form

$$
\left[u^{i} f^{i}\left(\eta^{i}\right)\right]_{t}=\left[u^{i} v^{i} f^{i}\left(\eta^{i}\right)\right]_{x},
$$

where $f^{i}\left(\eta^{i}\right)$ are arbitrary functions of their arguments. Although these conservation laws involve only $n$ (rather than the required $2 n$ ) arbitrary functions of one variable, their existence already implies the integrability of system (10), as well as of the corresponding system (6). This is a consequence of the following general proposition.

Proposition 1 Suppose that system (6) possesses $2 n$ conservation laws with functionally independent densities. Then, the system is integrable (and automatically possesses infinitely many conservation laws parametrised by $2 n$ arbitrary functions of one variable).

Proof This statement is a straightforward generalisation of the analogous fact known for diagonalisable systems: if system (2) possesses $n$ conservation laws with functionally independent densities, then it is semi-Hamiltonian (and, therefore, possesses infinitely many conservation laws parametrised by $n$ arbitrary functions of one variable), see (Sévennec 1994). In the present context, a proof can be summarised as follows. Note that all compatibility conditions of system (35) for conserved densities $h$ have the form

$$
\sum_{i} \varphi_{i} h_{r^{i}}+\sum_{i} \psi_{i} h_{\eta^{i}}=0
$$

where $\varphi_{i}$ and $\psi_{i}$ depend on the coefficients $a_{i}, b_{i}, a_{i j}, b_{i j}, c_{i j}, d_{i j}$ and partial derivatives thereof. We emphasise that the only 'free' second-order partial derivative $h_{r^{i}} \eta^{i}$ will not enter any of these conditions. Requiring that all compatibility conditions are satisfied identically, that is, $\varphi_{i}=\psi_{i}=0$, we recover the full set of integrability conditions (20)-(31). It remains to note that it is sufficient to require the existence of $2 n$ functionally independent densities $h$ to reach the same conclusion, indeed, any nontrivial compatibility condition imposes a linear homogeneous relation on the gradient of $h$, thus contradicting functional independence.

Setting in (37) $f^{i}=1$ and $f^{i}=\eta^{i}$, we obtain $2 n$ functionally independent densities $u^{i}$ and $u^{i} \eta^{i}$. Thus, by the above proposition, system (10), as well as the corresponding system (6), are integrable: all integrability conditions (20)-(31) are satisfied identically. This proof of integrability can be viewed as alternative to the one from Sect. 2.1. 
In fact, all conservation laws of system (10) can be found explicitly: the general conservation law has the form

$$
\left[\sum_{i=1}^{n} u^{i} \psi^{i}(\eta)+\sum_{i=1}^{n} \sigma^{i}\left(\eta^{i}\right)\right]_{t}=\left[\sum_{i=1}^{n} u^{i} v^{i} \psi^{i}(\eta)+\sum_{i=1}^{n} \tau^{i}\left(\eta^{i}\right)\right]_{x}
$$

here $\sigma^{i}\left(\eta^{i}\right)$ are arbitrary functions of one variable, the functions $\tau^{i}\left(\eta^{i}\right)$ can be recovered from the equations $\left(\tau^{i}\right)^{\prime}=\left(\sigma^{i}\right)^{\prime} \xi^{i}$ and the functions $\psi^{i}\left(\eta^{1}, \ldots, \eta^{n}\right)$ satisfy the equations $\psi_{, \eta^{j}}^{i}=\left(\sigma^{j}\right)^{\prime} \epsilon^{i j}, j \neq i$. The general conservation law (38) depends on $2 n$ arbitrary functions of one variable: $n$ functions $\sigma^{i}\left(\eta^{i}\right)$, plus extra $n$ functions coming from $\psi^{i}$. Setting in (38) $\sigma^{i}=0$ we recover conservation laws (37).

\section{Generalised Hodograph Formula}

Consider system (1) together with its commuting flow,

$$
u_{t}=V(u) u_{x} \text { and } u_{y}=W(u) u_{x}
$$

where $V(u)$ and $W(u)$ are $n \times n$ matrices (the commutativity conditions $u_{t y}=u_{y t}$ impose differential constraints on $V$ and $W$ ). Then the matrix relation

$$
W(u)=I x+V(u) t
$$

where $I$ is the $n \times n$ identity matrix, defines an implicit solution $u(x, t)$ of system (1) (Tsarev 1985, 1991). Note that, due to the commutativity conditions, only $n$ out of $n^{2}$ relations (39) will be functionally independent. Since any integrable system (1) possesses infinitely many commuting flows parametrised by $n$ arbitrary functions of one variable, formula (39) provides a general (multivalued) solution. For commuting systems (6) and (14), the hodograph formula (39) specialises to

$$
w^{i}(r, \eta)=x+v^{i}(r, \eta) t, \quad q^{i}(r, \eta)=p^{i}(r, \eta) t
$$

which is a system of $2 n$ implicit relations for the $2 n$ dependent variables $r, \eta$.

Proposition 2 Substituting into formula (40) $v^{i}, p^{i}$ from (12) and $w^{i}, q^{i}$ from (32), one obtains formula (13) for the general solution of system (6) corresponding to reduction (10) of the soliton gas equation:

$$
r^{i}=\frac{\varphi_{, \eta^{i}}^{i}-\left(\xi^{i}\right)^{\prime} t}{\mu^{i}}, \quad \varphi^{i}\left(\eta^{1}, \ldots, \eta^{n}\right)=x+\xi^{i}\left(\eta^{i}\right) t .
$$

Proof Using

$$
u^{i}=\sum_{k=1}^{n} \beta_{k i}, \quad v^{i}=\frac{1}{u^{i}} \sum_{k=1}^{n} \beta_{k i} \xi^{k}, \quad w^{i}=\frac{1}{u^{i}} \sum_{k=1}^{n} \beta_{k i} \varphi^{k},
$$


the relations $w^{i}=x+v^{i} t$ take the form

$$
\sum_{k=1}^{n} \beta_{k i} \varphi^{k}=x \sum_{k=1}^{n} \beta_{k i}+t \sum_{k=1}^{n} \beta_{k i} \xi^{k}
$$

equivalently,

$$
\sum_{k=1}^{n} \beta_{k i}\left(\varphi^{k}-x-\xi^{k} t\right)=0
$$

which gives the second half of the required equations due to non-degeneracy of matrix $\hat{\beta}$.

Similarly, using

$p^{i}=\frac{1}{u^{i}}\left(\sum_{k=1}^{n} \epsilon_{, \eta^{i}}^{k i}\left(v^{k}-v^{i}\right) u^{k}+\left(\xi^{i}\right)^{\prime}\right), \quad q^{i}=\frac{1}{u^{i}}\left(\sum_{k=1}^{n} \epsilon_{, \eta^{i}}^{k i}\left(w^{k}-w^{i}\right) u^{k}-r^{i} \mu^{i}+\varphi_{, \eta^{i}}^{i}\right)$,

the relations $q^{i}=p^{i} t$ take the form

$$
\sum_{k=1}^{n} \epsilon_{, \eta^{i}}^{k i}\left(w^{k}-w^{i}\right) u^{k}-r^{i} \mu^{i}+\varphi_{, \eta^{i}}^{i}=t\left(\sum_{k=1}^{n} \epsilon_{, \eta^{i}}^{k i}\left(v^{k}-v^{i}\right) u^{k}+\left(\xi^{i}\right)^{\prime}\right) .
$$

Using $w^{i}=x+t v^{i}$, both sums in the above relation cancel out, leaving

$$
-r^{i} \mu^{i}+\varphi_{, \eta^{i}}^{i}=t\left(\xi^{i}\right)^{\prime}
$$

the first half of the required equations.

\section{Examples}

In Sect. 5.1, we give some more details on integrable system (6) coming from reductions in the kinetic equation for soliton gas. In Sect. 5.2, we discuss hydrodynamic reductions of type (6) of the $3 D$ Mikhalev system.

\subsection{Reductions in the Kinetic Equation for Soliton Gas}

Let us begin with the simplest cases $n=1$ and $n=2$.

Case $n=1$. In this case, system (6) is a single $2 \times 2$ Jordan block,

$$
\begin{aligned}
& r_{t}=\xi r_{x}-r \xi^{\prime} \eta_{x}, \\
& \eta_{t}=\xi \eta_{x},
\end{aligned}
$$


recall that $\xi=-S(\eta)$. The general commuting flow of this system has the form

$$
\begin{aligned}
& r_{y}=\varphi r_{x}+\left(\mu r^{2}-\varphi^{\prime} r\right) \eta_{x} \\
& \eta_{y}=\varphi \eta_{x}
\end{aligned}
$$

where $\mu(\eta)$ and $\varphi(\eta)$ are two arbitrary functions. The corresponding generalised hodograph formula (40) simplifies to

$$
r=\frac{\varphi^{\prime}-\xi^{\prime} t}{\mu}, \quad \varphi(\eta)=x+\xi(\eta) t
$$

here the second relation specifies $\eta$ as an implicit function of $x$ and $t$; the first relation gives an explicit formula for $r$.

Case $n=2$. The corresponding $4 \times 4$ system (6) has two Jordan blocks; setting $\epsilon^{12}=\epsilon$ in (12) we have

$$
v^{1}=\frac{r^{2} \xi^{1}-\epsilon \xi^{2}}{r^{2}-\epsilon}, \quad v^{2}=\frac{r^{1} \xi^{2}-\epsilon \xi^{1}}{r^{1}-\epsilon}
$$

and

$$
p^{1}=\frac{\epsilon^{2}-r^{1} r^{2}}{r^{2}-\epsilon}\left(\frac{\xi^{1}-\xi^{2}}{r^{2}-\epsilon} \epsilon_{, \eta^{1}}+\left(\xi^{1}\right)^{\prime}\right), \quad p^{2}=\frac{\epsilon^{2}-r^{1} r^{2}}{r^{1}-\epsilon}\left(\frac{\xi^{2}-\xi^{1}}{r^{1}-\epsilon} \epsilon_{, \eta^{2}}+\left(\xi^{2}\right)^{\prime}\right)
$$

where $\xi^{1}=\xi^{1}\left(\eta^{1}\right), \xi^{2}=\xi^{2}\left(\eta^{2}\right)$ and $\epsilon\left(\eta^{1}, \eta^{2}\right)=G\left(\eta^{1}, \eta^{2}\right)$. Direct computation of the invariants from Sect. 2 gives

$$
\begin{aligned}
& a_{1}=a_{2}=0, \\
& b_{1}=\frac{2 r^{2}}{\epsilon^{2}-r^{1} r^{2}}, \quad b_{2}=\frac{2 r^{1}}{\epsilon^{2}-r^{1} r^{2}}, \\
& a_{12}=\frac{r^{1}-\epsilon}{r^{2}-\epsilon} \frac{\epsilon}{r^{1} r^{2}-\epsilon^{2}}, \quad a_{21}=\frac{r^{2}-\epsilon}{r^{1}-\epsilon} \frac{\epsilon}{r^{1} r^{2}-\epsilon^{2}}, \\
& b_{12}=\frac{\epsilon-r^{2} \frac{r^{1}-\epsilon}{r^{2}-\epsilon}}{r^{1} r^{2}-\epsilon^{2}} \epsilon_{, \eta^{2}}, \quad b_{21}=\frac{\epsilon-r^{1} \frac{r^{2}-\epsilon}{r^{1}-\epsilon}}{r^{1} r^{2}-\epsilon^{2}} \epsilon_{, \eta^{1}}, \\
& c_{12}=-\frac{r^{1}-\epsilon}{\left(r^{2}-\epsilon\right)^{2}} \epsilon_{, \eta^{1}}, \quad c_{21}=-\frac{r^{2}-\epsilon}{\left(r^{1}-\epsilon\right)^{2}} \epsilon_{, \eta^{2}}, \\
& d_{12}=\frac{r^{1}-\epsilon}{r^{2}-\epsilon} \epsilon_{, \eta^{1} \eta^{2}}+\frac{r^{1}-r^{2}}{\left(r^{2}-\epsilon\right)^{2}} \epsilon_{, \eta^{1}} \epsilon_{, \eta^{2}}, \quad d_{21}=\frac{r^{2}-\epsilon}{r^{1}-\epsilon} \epsilon_{, \eta^{1} \eta^{2}}+\frac{r^{2}-r^{1}}{\left(r^{1}-\epsilon\right)^{2}} \epsilon_{, \eta^{1}} \epsilon_{, \eta^{2}} ;
\end{aligned}
$$

note that the 'soliton velocities' $\xi^{1}$ and $\xi^{2}$ do not enter these expressions. In fact, modulo equivalence transformations (7), one can normalise $\xi^{i}\left(\eta^{i}\right) \equiv \eta^{i}$. Direct calculation shows that the corresponding $4 \times 4$ system is integrable: it satisfies all two-index integrability conditions (20)-(25). Note that the same conclusion is true in the more general case $G\left(\eta^{1}, \eta^{2}\right) \neq G\left(\eta^{2}, \eta^{1}\right)$, where the matrix $\epsilon$ is not symmetric. 
For $n=2$, equations (17), (18) for commuting flows are straightforward to solve. The general commuting flow is given by the formulae

$$
\begin{gathered}
w^{1}=\frac{r^{2} \varphi^{1}-\epsilon \varphi^{2}}{r^{2}-\epsilon}, \quad w^{2}=\frac{r^{1} \varphi^{2}-\epsilon \varphi^{1}}{r^{1}-\epsilon}, \\
q^{1}=\frac{r^{1} r^{2}-\epsilon^{2}}{r^{2}-\epsilon}\left(\frac{\varphi^{2}-\varphi^{1}}{r^{2}-\epsilon} \epsilon_{, \eta^{1}}+r^{1} \mu^{1}-\varphi_{, \eta^{1}}^{1}\right), \quad q^{2}=\frac{r^{1} r^{2}-\epsilon^{2}}{r^{1}-\epsilon}\left(\frac{\varphi^{1}-\varphi^{2}}{r^{1}-\epsilon} \epsilon_{, \eta^{2}}+r^{2} \mu^{2}-\varphi_{, \eta^{2}}^{2}\right) ;
\end{gathered}
$$

here $\varphi^{1}\left(\eta^{1}, \eta^{2}\right)$ and $\varphi^{2}\left(\eta^{1}, \eta^{2}\right)$ are two functions such that $\varphi_{, \eta^{2}}^{1}=\epsilon \mu^{2}$ and $\varphi_{, \eta^{1}}^{2}=$ $\epsilon \mu^{1}$, where $\mu^{1}\left(\eta^{1}\right)$ and $\mu^{2}\left(\eta^{2}\right)$ are arbitrary functions of the indicated arguments. Thus, the general commuting flow depends on four arbitrary functions of a single argument: $\mu^{1}\left(\eta^{1}\right), \mu^{2}\left(\eta^{2}\right)$, plus two more functions coming from the equations for $\varphi^{1}$ and $\varphi^{2}$. The corresponding generalised hodograph formula (40) simplifies to

$$
\begin{gathered}
r^{1}=\frac{\varphi_{, \eta^{1}}^{1}-\left(\xi^{1}\right)^{\prime} t}{\mu^{1}}, \quad r^{2}=\frac{\varphi_{, \eta^{2}}^{2}-\left(\xi^{2}\right)^{\prime} t}{\mu^{2}} \\
\varphi^{1}\left(\eta^{1}, \eta^{2}\right)=x+\xi^{1}\left(\eta^{1}\right) t, \quad \varphi^{2}\left(\eta^{1}, \eta^{2}\right)=x+\xi^{2}\left(\eta^{2}\right) t
\end{gathered}
$$

here the last two relations specify $\eta^{1}$ and $\eta^{2}$ as implicit functions of $x$ and $t$; the first two relations give explicit formulae for $r^{1}$ and $r^{2}$. This is a special case of the general formula (13).

For $n=2$, Eq. (35) for conserved densities is also straightforward to solve: a general conserved density has the form

$$
h=\frac{\left(\epsilon-r^{2}\right) \psi^{1}+\left(\epsilon-r^{1}\right) \psi^{2}}{r^{1} r^{2}-\epsilon^{2}}+\sigma^{1}\left(\eta^{1}\right)+\sigma^{2}\left(\eta^{2}\right)
$$

where $\sigma^{1}\left(\eta^{1}\right)$ and $\sigma^{2}\left(\eta^{2}\right)$ are arbitrary functions of the indicated arguments and $\psi^{1}\left(\eta^{1}, \eta^{2}\right)$ and $\psi^{2}\left(\eta^{1}, \eta^{2}\right)$ are two functions such that $\psi_{, \eta^{2}}^{1}=\left(\sigma^{2}\right)^{\prime} \epsilon$ and $\psi_{, \eta^{1}}^{2}=$ $\left(\sigma^{1}\right)^{\prime} \epsilon$. Thus, conserved densities depend on four arbitrary functions of one variable. Note that the above conserved density $h$ and the corresponding flux $g$ can be written in a simple form

$$
\begin{gathered}
h=u^{1} \psi^{1}+u^{2} \psi^{2}+\sigma^{1}\left(\eta^{1}\right)+\sigma^{2}\left(\eta^{2}\right) \\
g=u^{1} v^{1} \psi^{1}+u^{2} v^{2} \psi^{2}+\tau^{1}\left(\eta^{1}\right)+\tau^{2}\left(\eta^{2}\right),
\end{gathered}
$$

where $\left(\tau^{1}\right)^{\prime}=\left(\sigma^{1}\right)^{\prime} \xi^{1},\left(\tau^{2}\right)^{\prime}=\left(\sigma^{2}\right)^{\prime} \xi^{2}$. Formulae (42) and (44) generalise to the case of arbitrary $n$ in a straightforward way, leading to the general commuting flows (32) and the general conservation laws (38); in fact, this is the way formulae (32) and (38) were originally found.

Case $n>2$. Using computer algebra one can show that for $n=3$ and $n=4$, which correspond to $6 \times 6$ and $8 \times 8$ systems (6), all integrability conditions (20)-(31) are also satisfied identically (we do not present the explicit forms of $v^{i}$ and $p^{i}$ due to 
their complexity). A general proof (alternative to the ones given in Sects. 2 and 3 ) that works for arbitrary $n$ is based on the following explicit formulae for the coefficients $a_{i}, b_{i}, a_{i j}, b_{i j}, c_{i j}, d_{i j}$ :

$$
\begin{gathered}
a_{i}=0, \quad b_{i}=2 \beta_{i i}, \quad a_{i j}=\frac{u^{j}}{u^{i}} \beta_{j i}, \\
b_{i j}=\frac{\beta_{j i}}{u^{i}} \sum_{p} u^{p} \epsilon_{, \eta^{j}}^{p j}+\frac{u^{j}}{u^{i}} \sum_{p} \beta_{p i} \epsilon_{, \eta^{j}}^{j p}, \quad c_{i j}=\frac{u^{j}}{u^{i}} \sum_{p} \beta_{j p} \epsilon_{, \eta^{i}}^{p i}-\frac{u^{j}}{\left(u^{i}\right)^{2}} \beta_{j i} \sum_{p} u^{p} \epsilon_{, \eta^{i}}^{p i}, \\
d_{i j}=\frac{u^{j}}{u^{i}} \epsilon_{, \eta^{j}}^{j i} \eta^{i}+\frac{u^{j}}{u^{i}} \sum_{p} \sum_{q} \epsilon_{, \eta^{j}}^{j q} \beta_{q p} \epsilon_{, \eta^{i}}^{p i}+\frac{1}{u^{i}} \sum_{p} \beta_{j p} \epsilon_{, \eta^{i}}^{p i} \sum_{q} u^{q} \epsilon_{, \eta^{j}}^{q j} \\
-\frac{u^{j}}{\left(u^{i}\right)^{2}} \sum_{p} u^{p} \epsilon_{, \eta^{i}}^{p i} \sum_{q} \epsilon_{, \eta^{j}}^{j q} \beta_{q i}-\frac{\beta_{j i}}{\left(u^{i}\right)^{2}} \sum_{p} u^{p} \epsilon_{, \eta^{i}}^{p i} \sum_{q} u^{q} \epsilon_{, \eta^{j}}^{q j} .
\end{gathered}
$$

Since all these coefficients are expressed in terms of $u^{i}$ and $\beta_{i j}$, the verification of integrability conditions (20)-(31), which is a direct calculation, requires the following differentiation rules:

$$
\begin{array}{r}
\beta_{i j, r^{k}}=\beta_{i k} \beta_{k j}, \quad \beta_{i j, \eta^{k}}=\beta_{k j} \sum_{p} \beta_{i p} \epsilon_{, \eta^{k}}^{p k}+\beta_{i k} \sum_{p} \beta_{p j} \epsilon_{, \eta^{k}}^{k p}, \\
u_{, r^{j}}^{i}=u^{j} \beta_{j i}, \quad u_{, \eta^{j}}^{i}=\beta_{j i} \sum_{p} u^{p} \epsilon_{, \eta^{j}}^{p j}+u^{j} \sum_{p} \epsilon_{, \eta^{j}}^{j p} \beta_{p i} .
\end{array}
$$

Let us give a direct proof that formula (13) provides a general solution of reduction (10) of the soliton gas equation (which would not appeal to the generalised hodograph method).

Proposition 3 The general solution of system (6) corresponding to reduction (10) of the soliton gas equation is given by formula (13):

$$
r^{i}=\frac{\varphi_{, \eta^{i}}^{i}-\left(\xi^{i}\right)^{\prime} t}{\mu^{i}}, \quad \varphi^{i}\left(\eta^{1}, \ldots, \eta^{n}\right)=x+\xi^{i}\left(\eta^{i}\right) t
$$

here $\mu^{i}\left(\eta^{i}\right)$ are arbitrary functions of their arguments and the functions $\varphi^{i}\left(\eta^{1}, \ldots, \eta^{n}\right)$ satisfy the relations $\varphi_{, \eta^{j}}^{i}=\epsilon^{j i}\left(\eta^{i}, \eta^{j}\right) \mu^{j}\left(\eta^{j}\right), i \neq j$, no summation.

Proof Differentiating the last set of relations (13) by $x$ and $t$, we obtain

$\varphi_{, \eta^{i}}^{i} \eta_{x}^{i}+\sum_{j \neq i} \mu^{j} \epsilon^{j i} \eta_{x}^{j}=1+t\left(\xi^{i}\right)^{\prime} \eta_{x}^{i} \quad$ and $\quad \varphi_{, \eta^{i}}^{i} \eta_{t}^{i}+\sum_{j \neq i} \mu^{j} \epsilon^{j i} \eta_{t}^{j}=\xi^{i}+t\left(\xi^{i}\right)^{\prime} \eta_{t}^{i}$

respectively. Using $\varphi_{, \eta^{i}}^{i}-t\left(\xi^{i}\right)^{\prime}=r^{i} \mu^{i}$, we can rewrite these relations in the form

$$
r^{i} \mu^{i} \eta_{x}^{i}+\sum_{j \neq i} \mu^{j} \epsilon^{j i} \eta_{x}^{j}=1 \text { and } r^{i} \mu^{i} \eta_{t}^{i}+\sum_{j \neq i} \mu^{j} \epsilon^{j i} \eta_{t}^{j}=\xi^{i}
$$


Taking into account that $\epsilon^{i i}=r^{i}$, we come to

$$
\sum_{j=1}^{n} \mu^{j} \eta_{x}^{j} \epsilon^{j i}=1 \text { and } \sum_{j=1}^{n} \mu^{j} \eta_{t}^{j} \epsilon^{j i}=\xi^{i}
$$

Contracting these formulae (in the index $i$ ) with the inverse matrix $\beta_{i k}$ and using $\epsilon^{j i} \beta_{i k}=-\delta_{k}^{j}$, we obtain

$$
-\mu^{k} \eta_{x}^{k}=\sum_{i=1}^{n} \beta_{i k}=u^{k} \quad \text { and } \quad-\mu^{k} \eta_{t}^{k}=\sum_{i=1}^{n} \beta_{i k} \xi^{i}=u^{k} v^{k}
$$

which readily implies $\eta_{t}^{k}=v^{k} \eta_{x}^{k}$.

Similarly, differentiating $r^{i} \mu^{i}=\varphi_{, \eta^{i}}^{i}-t\left(\xi^{i}\right)^{\prime}$ by $x$ and $t$ we obtain (using $\varphi_{, \eta^{i} \eta^{j}}^{i}=$ $\left.\varphi_{, \eta^{j} \eta^{i}}^{i}=\epsilon_{, \eta^{i}}^{j i} \mu^{j}\right)$

$$
\begin{array}{r}
r_{x}^{i} \mu^{i}+r^{i}\left(\mu^{i}\right)^{\prime} \eta_{x}^{i}=\varphi_{, \eta^{i} \eta^{i}}^{i} \eta_{x}^{i}+\sum_{j \neq i} \epsilon_{, \eta^{i}}^{j i} \mu^{j} \eta_{x}^{j}-t\left(\xi^{i}\right)^{\prime \prime} \eta_{x}^{i}, \\
r_{t}^{i} \mu^{i}+r^{i}\left(\mu^{i}\right)^{\prime} \eta_{t}^{i}=\varphi_{, \eta^{i} \eta^{i}}^{i} \eta_{t}^{i}+\sum_{j \neq i} \epsilon_{, \eta^{i}}^{j i} \mu^{j} \eta_{t}^{j}-t\left(\xi^{i}\right)^{\prime \prime} \eta_{t}^{i}-\left(\xi^{i}\right)^{\prime},
\end{array}
$$

respectively. Equivalently,

$$
\begin{array}{r}
r_{x}^{i} \mu^{i}=\left(\varphi_{, \eta^{i} \eta^{i}}^{i}-r^{i}\left(\mu^{i}\right)^{\prime}-t\left(\xi^{i}\right)^{\prime \prime}\right) \eta_{x}^{i}+\sum_{j \neq i} \epsilon_{, \eta^{i}}^{j i} \mu^{j} \eta_{x}^{j}, \\
r_{t}^{i} \mu^{i}=\left(\varphi_{, \eta^{i} \eta^{i}}^{i}-r^{i}\left(\mu^{i}\right)^{\prime}-t\left(\xi^{i}\right)^{\prime \prime}\right) \eta_{t}^{i}+\sum_{j \neq i} \epsilon_{, \eta^{i}}^{j i} \mu^{j} \eta_{t}^{j}-\left(\xi^{i}\right)^{\prime} .
\end{array}
$$

Replacing $\eta_{t}^{i}$ by $v^{i} \eta_{x}^{i}$ and subtracting the first of the above equations (multiplied by $v^{i}$ ) from the second, we obtain:

$$
\left(r_{t}^{i}-v^{i} r_{x}^{i}\right) \mu^{i}=\sum_{j \neq i} \epsilon_{, \eta^{i}}^{j i} \mu^{j}\left(v^{j}-v^{i}\right) \eta_{x}^{j}-\left(\xi^{i}\right)^{\prime}
$$

To verify the equations $r_{t}^{i}=v^{i} r_{x}^{i}+p^{i} \eta_{x}^{i}$, it remains to show that the following identity holds:

$$
p^{i} \eta_{x}^{i} \mu^{i}=\sum_{j \neq i} \epsilon_{, \eta^{i}}^{j i} \mu^{j}\left(v^{j}-v^{i}\right) \eta_{x}^{j}-\left(\xi^{i}\right)^{\prime}
$$


This directly follows from the formula $\mu^{i} \eta_{x}^{i}=-u^{i}$, see (45); indeed, under this substitution the above formula reduces to

$$
p^{i} u^{i}=\sum_{j \neq i} \epsilon_{, \eta^{i}}^{j i}\left(v^{j}-v^{i}\right) u^{j}+\left(\xi^{i}\right)^{\prime}
$$

which identically coincides with (12).

Remark Reductions in the kinetic equation discussed in this paper satisfy the conditions $a_{i}=0$, which are equivalent to $v_{r^{i}}^{i}=0$. These can be seen as Jordan block analogues of the conditions of linear degeneracy which are known to prevent breakdown of classical solutions. Thus, Eq. (8) for soliton gas should be viewed as a linearly degenerate kinetic equation and is expected to have good 'regularity properties'.

\subsection{Reductions in the Mikhalev System}

Integrable systems of type (6), as well as integrable quasilinear systems (1) with a more general Jordan block structure, typically arise as hydrodynamic reductions of multi-dimensional linearly degenerate integrable PDEs. As a simple illustration, here we consider the linearly degenerate system

$$
m_{t}=n_{x}, \quad m_{y}=n_{t}-m n_{x}+n m_{x}
$$

which apparently first appeared in Mikhalëv (1992) in the context of Hamiltonian formalism of $\mathrm{KdV}$ type hierarchies and was subsequently investigated in the framework of multi-dimensional dispersionless integrability, see e.g. (Pavlov 2018; Ferapontov and Moss 2015). Hydrodynamic reductions are sought in the form

$$
m=m(r, \eta), \quad n=n(r, \eta)
$$

where the variables $r=\left(r^{1}, \ldots, r^{n}\right)$ and $\eta=\left(\eta^{1}, \ldots, \eta^{n}\right)$ satisfy a pair of commuting systems (6), (14). Remarkably, all such reductions can be found explicitly: they are parametrised by $n$ arbitrary functions of one variable. The substitution of (47) into (46) gives

$$
n_{r^{i}}=v^{i} m_{r^{i}}, \quad n_{\eta^{i}}=v^{i} m_{\eta^{i}}+p^{i} m_{r^{i}}
$$

as well as the dispersion relations,

$$
w^{i}=\left(v^{i}\right)^{2}-m v^{i}+n, \quad q^{i}=\left(2 v^{i}-m\right) p^{i} .
$$

Substituting (49) into commutativity conditions (15) and using (48), one obtains the relations

$$
v_{r^{i}}^{i}=m_{r^{i}}
$$


Substituting (49) into commutativity conditions (16) and using (48), one obtains the relations

$$
v_{r^{j}}^{i}=m_{r^{j}}, \quad v_{\eta^{j}}^{i}=m_{\eta^{j}}, \quad p_{r^{j}}^{i}=0, \quad p_{\eta^{j}}^{i}=0
$$

$i \neq j$. Furthermore, calculating consistency conditions of equations (48) we obtain

$$
m_{r^{i} r^{j}}=0, \quad m_{r^{i} \eta^{j}}=0, \quad m_{\eta^{i} \eta^{j}}=0
$$

$i \neq j$ (the last remaining consistency condition, $n_{r^{i} \eta^{i}}=n_{\eta^{i} r^{i}}$, will be taken into account later). Equations (50), (51) and (52) are straightforward to solve: they imply

$$
m=\sum_{k} f^{k}\left(r^{k}, \eta^{k}\right), \quad v^{i}=m+g^{i}\left(\eta^{i}\right), \quad p^{i}=p^{i}\left(r^{i}, \eta^{i}\right) .
$$

Without any loss of generality, modulo equivalence transformations (7), one can set $m=\sum_{k} r^{k}, v^{i}=m+\eta^{i}$. Then the last remaining consistency condition, $n_{r^{i} \eta^{i}}=n_{\eta^{i} r^{i}}$, implies $p_{r^{i}}^{i}=1$, which we will solve in the form $p^{i}=r^{i}+\left(s^{i}\left(\eta^{i}\right)\right)^{\prime}$. Ultimately, we arrive at the following formulae:

$$
\begin{aligned}
& m=\sum_{k} r^{k}, \quad n=\frac{1}{2} m^{2}+\sum_{k} r^{k} \eta^{k}+\sum_{k} s^{k}\left(\eta^{k}\right), \quad v^{i}=m+\eta^{i} \\
& p^{i}=r^{i}+\left(s^{i}\left(\eta^{i}\right)\right)^{\prime}
\end{aligned}
$$

here $s^{k}\left(\eta^{k}\right)$ are arbitrary functions of their arguments. Integrability of these reductions can be verified by direct calculation.

Acknowledgements We thank A. Bolsinov, G. El, and D. Tunitsky for clarifying discussions. We also thank the referees for useful comments. The research of EVF was supported by a grant from the Russian Science Foundation No. 21-11-00006, https://rscf.ru/project/21-11-00006/. MVP was supported by the Ministry of Science and Higher Education of the Russian Federation (Agreement No. 075-02-2021-1748).

Open Access This article is licensed under a Creative Commons Attribution 4.0 International License, which permits use, sharing, adaptation, distribution and reproduction in any medium or format, as long as you give appropriate credit to the original author(s) and the source, provide a link to the Creative Commons licence, and indicate if changes were made. The images or other third party material in this article are included in the article's Creative Commons licence, unless indicated otherwise in a credit line to the material. If material is not included in the article's Creative Commons licence and your intended use is not permitted by statutory regulation or exceeds the permitted use, you will need to obtain permission directly from the copyright holder. To view a copy of this licence, visit http://creativecommons.org/licenses/by/4.0/.

\section{Appendix: Derivation of System (10)}

Although delta-functional reduction in the kinetic equation for soliton gas was first obtained in Pavlov et al. (2012), to make the paper more self-contained, below we present a short derivation of system (10). 
Substituting ansatz (9),

$$
f(\eta, x, t)=\sum_{i=1}^{n} u^{i} \delta\left(\eta-\eta^{i}\right)
$$

into the first equation (8),

$$
f_{t}+(s f)_{x}=0
$$

we obtain

$$
\sum_{i=1}^{n}\left(u_{t}^{i}+s u_{x}^{i}+s_{x} u^{i}\right) \delta\left(\eta-\eta^{i}\right)-\sum_{i=1}^{n} u^{i}\left(\eta_{t}^{i}+s \eta_{x}^{i}\right) \delta^{\prime}\left(\eta-\eta^{i}\right)=0
$$

Multiplying this equality by a test function $\varphi(\eta)$ and integrating over $\eta$ (using integration by parts to eliminate $\delta^{\prime}$ ) gives

$$
\begin{array}{r}
\sum_{i=1}^{n}\left(u_{t}^{i}+s\left(\eta^{i}, x, t\right) u_{x}^{i}+\right. \\
\left.u^{i} s_{x}\left(\eta^{i}, x, t\right)+u^{i} s_{\eta}\left(\eta^{i}, x, t\right) \eta_{x}^{i}\right) \varphi\left(\eta^{i}\right) \\
+\sum_{i=1}^{n} u^{i}\left(\eta_{t}^{i}+s\left(\eta^{i}, x, t\right) \eta_{x}^{i}\right) \varphi^{\prime}\left(\eta^{i}\right)=0 .
\end{array}
$$

Introducing the notation $v^{i}=-s\left(\eta^{i}, x, t\right)$ and equating to zero the coefficient at $\varphi\left(\eta^{i}\right)$, we obtain $u_{t}^{i}=\left(u^{i} v^{i}\right)_{x}$. Similarly, equating to zero the coefficient at $\varphi^{\prime}\left(\eta^{i}\right)$ gives $\eta_{t}^{i}=v^{i} \eta_{x}^{i}$. Thus, we have recovered equations (10).

Substituting ansatz (9) into the second equation (8),

$$
s(\eta)=S(\eta)+\int_{0}^{\infty} G(\mu, \eta) f(\mu)[s(\mu)-s(\eta)] d \mu,
$$

gives

$$
s(\eta, x, t)=S(\eta)+\sum_{i=1}^{n} G\left(\eta^{i}, \eta\right) u^{i}\left[s\left(\eta^{i}, x, t\right)-s(\eta, x, t)\right]
$$

Setting $\eta=\eta^{k}$ and using $v^{i}=-s\left(\eta^{i}, x, t\right)$, we obtain $n$ linear relations for $v^{i}$,

$$
v^{k}=-S\left(\eta^{k}\right)+\sum_{i \neq k} G\left(\eta^{i}, \eta^{k}\right) u^{i}\left[v^{i}-v^{k}\right]
$$

which are identical to (11) under the interchange of indices $i \leftrightarrow k$. 


\section{Concluding Remarks}

The approach to hydrodynamic integrability developed in this paper can be generalised to the whole class of non-semisimple quasilinear systems (1) whose matrix $V$ has block-diagonal structure such that:

- each diagonal block has upper-triangular Toeplitz form as in Xue and Ferapontov (2020), with the unique eigenvalue; here is a $3 \times 3$ Toeplitz matrix with eigenvalue $v$ :

$$
\left(\begin{array}{lll}
v & p & q \\
0 & v & p \\
0 & 0 & v
\end{array}\right)
$$

- different blocks have different eigenvalues.

Matrices of this type (known as gl-regular) form a commutative family, and the corresponding systems (1) automatically have zero Haantjes tensor. Commuting flows of such systems necessarily have Toeplitz blocks of the same sizes. An $n \times n$ system of this type should be called integrable if it possesses a hierarchy of hydrodynamic commuting flows/conservation laws parametrised by $n$ arbitrary functions of one variable. In this connection, let us mention that a criterion for block-diagonalisability of quasilinear systems was obtained in Bogoyavlenskij (2006) (theorems 1, 2).

Finally, it would be interesting to consider continuum versions of ansatz (9), system (10), and its general solution (13), with an ultimate goal of establishing integrability of the full kinetic equation (8).

\section{References}

Bogoyavlenskij, O.I.: Block-diagonalizability problem for hydrodynamic type systems. J. Math. Phys. 47(6), 063502 (2006)

Doyon, B., Yoshimura, T.: Soliton gases and generalized hydrodynamics, J.-S. Caux. Phys. Rev. Lett. 120, 045301 (2018)

Dubrovin, B.A., Novikov, S.P.: Hydrodynamics of weakly deformed soliton lattices: differential geometry and Hamiltonian theory. Russ. Math. Surv. 44, 35-124 (1989)

El, G.A., Kamchatnov, A.M., Pavlov, M.V., Zykov, S.A.: Kinetic equation for a soliton gas and its hydrodynamic reductions. J. Nonlinear Sci. 21(2), 151-191 (2011)

El, G.A.: The thermodynamic limit of the Whitham equations. Phys. Lett. A 311(4-5), 374-383 (2003)

El, G.A., Kamchatnov, A.M.: Kinetic equation for a dense soliton gas. Phys. Rev. Lett. 95, 204101 (2005)

El, G.A., Tovbis, A.: Spectral theory of soliton and breather gases for the focusing nonlinear Schrödinger equation. Phys. Rev. E 101, 052207 (2020)

Ferapontov, E.V., Moss, J.: Linearly degenerate PDEs and quadratic line complexes. Commun. Anal. Geom. 23(1), 91-127 (2015)

Kodama, Yu., Konopelchenko, B.G.: Confluence of hypergeometric functions and integrable hydrodynamictype systems. Theor. Math. Phys. 188(3), 1334-1357 (2016)

Konopelchenko, B.G., Ortenzi, G.: Parabolic regularization of the gradient catastrophes for the BurgersHopf equation and Jordan chain. J. Phys. A 51(27), 275201 (2018)

Mikhalëv, V.G.: Hamiltonian formalism of Korteweg-de Vries-type hierarchies. Funct. Anal. Appl. 26(2), 140-142 (1992)

Pavlov, M.V.: Integrability of exceptional hydrodynamic-type systems. Proc. Steklov Inst. Math. 302(1), 325-335 (2018) 
Pavlov, M.V., Taranov, V.B., El, G.A.: Generalized hydrodynamic reductions of the kinetic equation for a soliton gas. Theor. Math. Phys. 171(2), 675-682 (2012)

Serre, D.: Systems of Conservation Laws. 1. Hyperbolicity, Entropies. Shock Waves. Cambridge University Press, Cambridge (1999)

Serre, D.: Systems of Conservation Laws. 2. Geometric Structures, Oscillations, and Initial-Boundary Value Problems. Cambridge University Press, Cambridge (2000)

Sévennec, B.: Géométrie des systèmes hyperboliques de lois de conservation, Mémoire (nouvelle série) N56, Supplément au. Bull. Soc. Math. France 122, 1-125 (1994)

Tsarev, S.P.: Poisson brackets and one-dimensional Hamiltonian systems of hydrodynamic type. Sov. Math. Dokl. 31, 488-491 (1985)

Tsarev, S.P.: The geometry of Hamiltonian systems of hydrodynamic type. The generalized hodograph method. Math. USSR Izvestiya 37, 397-419 (1991)

Xue, L., Ferapontov, E.V.: Quasilinear systems of Jordan block type and the mKP hierarchy. J. Phys. A Math. Theor. 53, 205202 (2020)

Zakharov, V.E.: Kinetic equation for solitons. Sov. Phys. JETP 33, 538-541 (1971)

Publisher's Note Springer Nature remains neutral with regard to jurisdictional claims in published maps and institutional affiliations. 